\title{
CLASSIFICATION OF COMPACT OBJECTS: QSS, QSOS, N-TYPE AND COMPACT GALAXIES, SEYFERT AND GALACTIC NUCLEI
}

\author{
W. W. MORGAN \\ Yerkes Observatory, Williams Bay, Wis., U.S.A.
}

\begin{abstract}
Some methods currently in use for the classification of the optical forms of the 'compact' galaxies and quasi-stellar objects are reviewed. It is shown that the category 'Seyfert Galaxy' is basically a spectroscopic (rather than a form) classification.

An optical form-classification is described which is, in principle, identical with published classification criteria for QSO, N-type, and compact objects. The importance of maintaining rigid formstandards is emphasized.
\end{abstract}

\section{Introduction}

The forms of compact extragalactic objects differ from ordinary spirals and ellipticals; and it is not possible to interpolate satisfactorily the compact objects in the Hubble or Yerkes sequences of standard types. The optical forms observed for galaxies having eruptive nuclei have their own peculiar shapes, which require a special morphology.

This has led to definitions of certain categories. For example, Sandage (1967) has given a short definition and distinction between the QSS and the N-type galaxies:

"These systems, called $\mathrm{N}$-galaxies, have some resemblance in optical appearance to quasi-stellar sources (QSS) in that most of the light comes from the pointlike nucleus. However, QSS and $\mathrm{N}$-galaxies differ; all $\mathrm{N}$-galaxies show an outer nebulous envelope on plates taken with the 48-in. Schmidt, while all QSS are indistinguishable from stars..."

In the same paper, Sandage showed that the N-galaxies and QSS are cleanly separated in the UBV two-color plot - and that the radio-quiet QSO occupy the area defined by the QSS.

Later, C. R. Lynds (1968) published a discussion of the forms and spectra of certain galaxies classified as $\mathrm{N}$-type. He showed that the object Braccesi 234 , while completely stellar in appearance, falls in the N-type domain of the two-color plot. This object has a low redshift, and sharp emission hydrogen and forbidden lines. He also states that there are galaxies classified as $\mathrm{N}$-type that show no emission lines, but that do contain $\mathrm{H}$ and $\mathrm{K}$, and the G-band in absorption. The high level of the critical approach used by Lynds on both form and spectral characteristics sets a standard for similar investigations of the future.

The basic problem, then, is to examine and describe the forms and spectra of the compact objects (QSO, N, compact, Seyfert) with the greatest individual precision while at the same time constructing the most useful groupings of the objects in some morphological scheme. 


\section{Comments on the Classification of QSO, N-Type and Seyfert Galaxies}

There seems to be general agreement on the definition of quasi-stellar objects (G. R. Burbidge, 1970): "QSOs are defined as a class of objects which have a star-like appearance on direct plates and have very large redshifts in their spectra". However, two of the three first discoveries (Matthews and Sandage, 1963) were noted as having "exceedingly faint wisps of nebulosity" associated with the star-like images. The surveys of Sandage and Véron (1965) and Braccesi et al. (1968) furnished spectrophotometric criteria for candidates for non-radio QSOs.

The N-galaxies have been defined (Morgan, 1958) as "systems having small brilliant nuclei superposed on a considerably fainter background"; later, $\mathrm{N}$-type radio-galaxies were defined by Matthews et al. (1964) as "galaxies having brilliant star-like nuclei containing most of the luminosity of the system. A faint nebulous envelope of small visible extent is observed".

As has been noted by a number of investigators, the above-mentioned definitions follow rather closely the earlier comments by Seyfert on the forms of the nuclear region of the galaxies in his classical paper (Seyfert, 1943): “... their most consistent characteristic being an exceedingly luminous stellar or semi-stellar nucleus which contains a relatively large percentage of the total light of the system".

These similar definitions complicate the classification problem; and if each is literally applicable there would seem to be no reason for the introduction of the $\mathrm{N}$-category. However, re-examination of the optical form of the twelve original Seyfert galaxies indicates that Seyfert's optical description quoted above does not hold for some of the galaxies in his paper. Burbidge et al. (1963) showed that four of the twelve galaxies listed by Seyfert (NGC 2782, 3077, 4258, and 6814) do not satisfy their reformulation of Seyfert's criteria:

"(i) it has a small bright nucleus.

(ii) the spectrum of the nucleus contains emission features which are not normally seen in the spectra of galaxies. Some of these should be lines indicating higher excitation than that required to excite [O III] and [NeIII]...

(iii) the emission features; or at least the hydrogen emission lines, must be of great width, which, if interpreted as Doppler motions, corresponds to velocities in the range \pm 500 to $\pm 4250 \mathrm{~km} \mathrm{~s}^{-1}$, according to Seyfert"'.

Recent observations by Nolan Walborn of the forms of seven of the eight remaining galaxies in Seyfert's list with the new 41-in. reflector at the Yerkes Observatory indicate that two others (NGC 1275 and 3516) do not accord with Seyfert's optical criterion: also, the small nucleus of NGC 1068 can hardly be said to contain "a relatively large percentage of the total light of the system". From the optical forms of the eight currently accepted members of Seyfert's group, only five (NGC 3227, 4051, 4151,5548 , and 7469) satisfy all optical conditions for membership in the group (the data for NGC 3227 were taken from Rubin and Ford (1968)).

We now have two questions which require comment: Does the ' $N$ type' classifi- 
cation differ from the 'Seyfert' classification? And: What is the nature of the Seyfert classification?

The N-type classification is based on a certain appearance of the optical image. The description of the class is formulated for images containing a relatively small number of picture elements, for the sake of use over large ranges in distance without systematic error. When the number of picture elements is increased (through proximity or the use of a larger telescope) it is seen that many $\mathrm{N}$-galaxies are spiral in nature; the class ' $N$ ', therefore, includes a subclass 'NS'. Under these terms, one (NGC 5548) of the eight Seyfert galaxies is an outstanding example of the $\mathrm{N}$-type, while two (NGC 1275 and 3516) are different - and could not properly be described as of N-type. We therefore have the following situation: The form of one of the original Seyfert galaxies can be considered as the prototype for class $\mathrm{N}$, as defined above; certain other of the Seyfert galaxies could not properly be classified as $\mathbf{N}$, while the forms of still others are intermediate in appearance. The 'Seyfert' category does not define a particular form-class; it does define, however, a rather particular combination of spectroscopic characteristics. If we oversimplify somewhat the latter, we can describe a spectroscopic category which has some characteristics of a 'natural group': strong, relatively narrow $N_{1}$ and $N_{2}$ emissions combined with very broad emission Balmer lines.

If we apply the term 'Seyfert' as a spectroscopic description, then we avoid the fundamental ambiguity between categories 'Seyfert', 'N', and 'quasi-stellar object'. The 'Seyfert' classification can be used unambiguously as a spectroscopic class; the types 'QSO', 'N', and 'C' (to be discussed below) then furnish an unambiguous group of form-categories. The 'Seyfert spectrum' category (as suggested recently by Arp (1970)), then also becomes unambiguous in use and can be applied as information to many C-type, N-type, and QSO objects - in the sense of additional, rather than competing data. When considered as a spectral, rather than a form, classification, the Seyfert types as illustrated in the spectral tracings in Seyfert's paper are found to be present in members of all three basic form categories (QSO, N, Compact). In addition, the varied spectroscopic characteristics described by Lynds (and later by Sargent and others), furnish material of unique value for astrophysical investigation and for possible relationships between luminosity on one hand, and form and spectrum on the other.

\section{The Form-Classification of the Zwicky, Markarian and Haro Objects}

The most careful consideration of the nature of objects classified as 'compact' by Zwicky has been carried out by Sargent (1970b). The definitions given below are based on his conclusions. Zwicky (1964) has defined a compact galaxy in these terms:

"We call compact galaxies those which can just be distinguished from stars on plates taken with the Palomar 48-in. telescope and which have diameters of 2 " -5 "..",

"We shall call a galaxy moderately compact if its image, on photographs taken with the 18-in. Palomar Schmidt telescope, can just barely be distinguished from stars of the same apparent brightness. These systems have diameters of about $5^{\prime \prime}-10^{\prime \prime} \ldots$, 
Sargent summarizes the results of his own observations of a number of the galaxies in Zwicky's lists as follows:

"An inspection of the Palomar Sky Survey prints reveals that while, in accord with the definition above, the majority of the objects are small, a considerable minority are extended, often with bright knots, jets, distorted spiral arms, bridges to other nearby galaxies, rings, or some such unusual appendage. These, presumably, are the eruptive and post-eruptive galaxies and the galaxies with compact parts... The feature that is common to the objects in Zwicky's lists is that all have a region of high surface brightness."

From the above it can be seen that a simple, precise form-classification for such a varied collection of remarkable objects is impossible. In his beautiful discussion Sargent has recognized this, and has proceeded to give careful, individual descriptions of the forms and spectra of the objects observed. Such a procedure is a necessity in laying the groundwork for a later form-classification, and a detailed spectral classification as well.

At the present time, the basic spectroscopic approach used by Sargent for separation into astrophysical categories is certainly the most fruitful general procedure. The forms of a certain number of the Zwicky objects can be classified on the form system described below, but they constitute a minority of the objects observed by Sargent. Warning should be given against an overclassification of the Zwicky optical forms by relaxing or straining the criteria; such a procedure would further complicate, rather than clear, the present situation.

The seventy galaxies listed by Markarian possess abnormally strong ultraviolet continua, as observed by objective prism camera with a dispersion of $\sim 1800 \AA \mathrm{mm}^{-1}$ near $\mathrm{H} \gamma$. Most of these galaxies are compact, with sharp, star-like nuclei. The selection, then, is from a spectroscopic criterion, and differs from that of Zwicky.

E. Khachikian has summarized the results of joint investigations of forty of these objects (Khachikian, 1968; Weedman and Khachikian, 1968, 1969). The range in spectroscopic characteristics is great, represented by the five groups:

(1) Narrow emission and absorption lines;

(2) Narrow, strong emission lines only;

(3) Strong and diffuse emission lines; $N_{1}, N_{2}$ much stronger than the hydrogen lines;

(4) No strong emission lines;

(5) Very broad hydrogen emission lines, typical of Seyfert galaxies.

Sargent notes that the Zwicky emission-line compact objects resemble the Markarian galaxies and the Haro (1956) galaxies.

In another paper, Sargent (1970a) discusses the nature of the Markarian galaxies in terms of the latter's classification and his own new spectrograms. The Markarian classification of the sharpness and strength of the ultraviolet continuum has resulted in the discovery of four new Seyfert-type spectra (Markarian 50, 69, 9, 10). The first two of these are described by Sargent as "structureless, blue, compact objects". The last two are classified below. From the thirty galaxies observed by Sargent the conclusion is drawn that the Markarian objects comprise a non-homogeneous group. 
The Haro galaxies were selected on the basis of excesses in $U$, of $U, B, V$ images on Tonantzintla Schmidt plates. An investigation by DuPuy (1968a, 1968b) showed that on the Palomar Sky Survey prints almost all of the Haro objects show "... structural distortions or highly irregular forms". From this it can be concluded that classification of their optical forms will be affected by the same difficulties as in the cases of the Zwicky and Markarian objects; and in all three catalogues the spectroscopic approach seems to be the most promising at present.

\section{A Compilation of Criteria for Form-Classification: A Catalogue of Form-Types}

We make use of three basic form classes: $\mathrm{Q}, \mathrm{N}, \mathrm{C}$ : with two additional subclasses: $\mathrm{N}-$ and NS:

Q: Objects having a star-like appearance on direct plates, with large redshifts.

$\mathrm{N}$ : Galaxies having small, brilliant nuclei containing a considerable fraction of the total luminosity, superposed on a considerably fainter main body.

C: Small, high surface-brightness galaxies which are slightly resolved on medium and large-scale photographs. (Adapted from definition given by Zwicky to Arp (private communication, 1970)).

To this must be added (to minimize selection effects with distance), the following: "structureless, blue, compact objects" (Sargent, 1970a).

$\mathrm{N}-$ : Less pronounced $\mathrm{N}$-galaxies. (This is included to minimize selection effects with resolution or distance.)

NS: N-galaxies having well-developed spiral arms. (This is included to minimize selection effects with resolution or distance.)

Some standards for form classes are given below:

TABLE I

Object
NGC 5548
3C 48
III Zw 2
3C 120
Markarian 9
Zw $0039.5+4003$
NGC 7469
B $264^{a}$
Markarian 10
NGC 7469
NGC 4051
NGC 3516
Ton 256
B 264 ${ }^{\text {b }}$

a From 200 -in. print
b From 48 -in. red print

\section{Source of illustration}

Walborn, Yerkes 41-in.

Sandage and Miller (1966)

Arp (1968)

Arp (1968)

Khachikian (1968)

Zwicky et al. (1970)

Walborn, Yerkes 41-in., low resolution

Arp (1970)

Khachikian (1968)

Walborn, Yerkes 41-in., high resolution; Burbidge et al. (1963)

Morgan (1958)

Walborn, Yerkes 41-in.

Arp (1970)

Arp (1970) 


\section{Conclusion}

One of the most remarkable characteristics of the Zwicky and Markarian objects is their great variety of appearance on large-scale photographs, as shown by Sargent. This characteristic makes form-classification especially difficult; but the fact of the existence of this variety is a discovery of great importance. It is to be hoped that the remainder of these objects can be observed, both spectroscopically and by direct photography.

The relationship of the $\mathrm{N}$-type galaxies to the quasi-stellar objects is of crucial importance. A recent paper by Komberg and Ozernoy (1970) defines an epoch in this field and suggests many fruitful subjects for future research.

A remarkable and finely balanced general summary of the field of nuclei of galaxies has just been published by G. R. Burbidge (1970). This accurately delineates the state of the field in early 1970, and raises many fundamental questions for further investigation.

\section{Acknowledgements}

I am indebted for discussions of the classification of Seyfert and compact galaxies to Drs V. A. Ambartsumian, W. L. W. Sargent, and J. B. Oke; Drs Halton Arp, E. M. Burbidge, Sargent, and J. N. Bahcall sent preprints of work in this field. I am especially indebted to Dr G. R. Burbidge for his preprint (Burbidge, 1970). Photographic prints of B 264 and Ton 256 from Dr Arp made possible the classification of these objects. The plates of NGC 3516, 4051, 4151, 5548 and 7469 were taken by Mr. Nolan Walborn. Mr. J. W. Tapscott carried out necessary photographic work with distinction. The investigation was supported by a grant from the National Science Foundation.

\section{References}

Arp, H. C.: 1968, Astrophys. J. 152, 1101.

Arp, H. C.: 1970, Astrophys. J., in press.

Braccesi, A., Lynds, C. R., and Sandage, A. R.: 1968, Astrophys. J. Letters 152, L105.

Burbidge, G. R.: 1970, Ann. Rev. Astron. Astrophys. 8, 369.

Burbidge, G. R., Burbidge, E. M., and Prendergast, K. H.: 1963, Astrophys. J. 137, 1022.

Haro, G.: 1956, Bol. Obs. Tonantzintla Tacubaya 2, 8.

Khachikian, E.: 1968, Astron. J. 73, 891.

Komberg, B. V. and Ozernoy, L. M.: 1970, Astrophys. Space Sci. 7, 31.

Lynds, C. R.: 1968, Astron. J. 73, 88.

Matthews, T. A., Morgan, W. W., and Schmidt, M.: 1964, Astrophys. J. 140, 35.

Matthews, T. A. and Sandage, A. R.: 1963, Astrophys. J. 138, 30.

Morgan, W. W.: 1958, Publ. Astron. Soc. Pacific 70, 364.

du Puy, D.: 1968a, Astron. J. 73, 882.

du Puy, D.: 1968b, Publ. Astron. Soc. Pacific 80, 29.

Rubin, V. C. and Ford, W. K.: 1968, Astrophys. J. 154, 431.

Sandage, A. R.: 1967, Astrophys. J. Letters 150, L9.

Sandage, A. R. and Miller, W. C.: 1966, Astrophys. J. 144, 1238.

Sandage, A. R. and Véron, P.: 1965, Astrophys. J. Letters 142, L412.

Sargent, W. L. W.: 1970a, Astrophys. J. 159, 765.

Sargent, W. L. W.: 1970b, Astrophys. J. 160, 405. 
Seyfert, C.: 1943, Astrophys. J. 97, 28.

Weedman, D. W. and Khachikian, E.: 1968, Astrofizika 4, 587.

Weedman, D. W. and Khachikian, E.: 1969, Astrofizika 5, 113.

Zwicky, F.: 1964, Astrophys. J. 140, 1467.

Zwicky, F., Oke, J. B., Neugebauer, G., Sargent, W. L. W., and Fairall, A. P.: 1970, Publ. Astron. Soc. Pacific 82, 93.

\section{Discussion}

Miley: Radio quasars can be divided into two main classes according to their radio properties - those such as $3 \mathrm{C} 273$ with an active component radio nucleus coincident with the optical QSO, and those such as $3 \mathrm{C} 47$ with similar radio components located symmetrically about the optical QSO. These latter quasars tend to have steeper radio spectra which do not vary with time. It will be very interesting to discover whether the optical type can tell us anything about these two radio classes.

Tifft: NGC 4303 is a blue nuclear system photometrically. This concurs nicely with your note on masking of the blue spectrum by a blue continuum source. NGC 4501 is a red nuclear system photometrically. This concurs with an enriched dwarf system. There is obviously a fine case for smooth continuity between 'normal' and 'abnormal' nuclei. For example Seyfert - photometrically abnormal nuclei - normal.

Arp: The value of a precise and completely empirical classification on the basis of form is that we can now ask what is the transition between the star-like QSOs on the one hand and compact and $\mathrm{N}$-galaxies on the other hand. One kind of transition could take place by an increasingly larger and brighter disk appearing around a stellar nucleus. Another kind of transition could take place if QSOs and compact objects had an increasing number of jets emerging from the nucleus until they formed the disk of an N-galaxy. Possible examples of this last kind of transition would be 3C 48 and 3C 120 .

Wlérick: Just a remark concerning the galaxy NGC 4051. I am a bit shy to make it after the publication of the wonderful photograph of NGC 4151 taken with the Stratoscope II. Still I would like to report that on photographs taken with the electron camera at the Haute Provence Observatory in March 1968, the 'nucleus' of NGC 4051 appears elongated, in the V and B colors. Although the 'seeing' was medium, between 1" and 2", all the plates are consistent with a model in which there is one true 'nucleus', nearly stellar, and two relatively strong 'jets' that on the photographs are embedded in the apparent elliptical nucleus; the two-dimensional nebulosity starts beyond this nearly onedimensional structure.

Dent: In this classification scheme, how would quasi-stellar objects with jets be classified? Would they be N-type, since the jets are, after all, a nebulosity? I'm thinking of 3C 273 and 3C 279 as examples.

Morgan: I would not classify 3C 273 as an N-type galaxy from the isolated jet which has been observed. If later observations should reveal a nebulous envelope, the classification should be re-examined.

Wray: It appears to me that the general problem of direct observation of these types of objects would be a rewarding area of work for someone possessing an electronographic camera. The large dynamic range would provide an improved capacity to record both the relatively bright nucleus and the fainter amorphous outer regions in a single exposure, while the relatively low noise would permit maximum information retention for the nearly stellar objects and would also improve the possibility of achieving at least a limited deconvolution of the point image spread caused by the atmospheric degradation and image brightness.

Khachikian: What do you think the lower limit to the width of a galaxy's H-lines is, to call it a Seyfert type?

Morgan: This is a matter for later decision by the spectroscopists. The extreme spectra are easy to classify, but there will also be borderline cases. 